\title{
Copy number and nucleotide variation of the LILR family of myelomonocytic cell activating and inhibitory receptors
}

\author{
María R. López-Álvarez • Des C. Jones • Wei Jiang • \\ James A. Traherne $\cdot$ John Trowsdale
}

Received: 12 September 2013 / Accepted: 24 October 2013 / Published online: 21 November 2013

(C) The Author(s) 2013. This article is published with open access at Springerlink.com

\begin{abstract}
Leukocyte immunoglobulin-like receptors (LILR) are cell surface molecules that regulate the activities of myelomonocytic cells through the balance of inhibitory and activation signals. LILR genes are located within the leukocyte receptor complex (LRC) on chromosome 19q13.4 adjacent to KIR genes, which are subject to allelic and copy number variation (CNV). LILRB3 (ILT5) and LILRA6 (ILT8) are highly polymorphic receptors with similar extracellular domains. LILRB3 contains inhibitory ITIM motifs and LILRA6 is coupled to an adaptor with activating ITAM motifs. We analysed the sequences of the extracellular immunoglobulin domain-encoding regions of LILRB3 and LILRA6 in 20 individuals, and determined the copy number of these receptors, in addition to those of other members of the LILR family. We found 41 polymorphic sites within the extracellular domains of LILRB3 and LILRA6. Twenty-four of these sites were common to both receptors. LILRA6, but not LILRB3,
\end{abstract}

M.R. López-Álvarez and D.C. Jones contributed equally to this work.

Electronic supplementary material The online version of this article (doi:10.1007/s00251-013-0742-5) contains supplementary material, which is available to authorized users.

M. R. López-Álvarez · D. C. Jones · W. Jiang · J. A. Traherne · J. Trowsdale

Department of Pathology, University of Cambridge, Tennis Court Road, Cambridge CB2 1QP, UK

M. R. López-Álvarez $\cdot$ W. Jiang · J. A. Traherne · J. Trowsdale Cambridge Institute for Medical Research, University of Cambridge, Cambridge Biomedical Campus, Wellcome Trust/MRC Building, Hills Road, Cambridge CB2 OXY, UK

J. Trowsdale $(\bowtie)$

Immunology Division, Pathology Department,

University of Cambridge, Cambridge CB2 1QP, UK

e-mail: jt233@cam.ac.uk exhibited CNV. In 20 out of 48 human cell lines from the International Histocompatibility Working Group, LILRA6 was deleted or duplicated. The only other LILR gene exhibiting genomic aberration was LILRA3, in this case due to a partial deletion.

Keywords LILR $\cdot$ ILT $\cdot$ CNV $\cdot$ Haplotype

\section{Introduction}

Leukocyte immunoglobulin (Ig)-like receptors (LILR; also termed LIR or ILT) are mainly expressed on the surface of myelomonocytic cells (Brown et al. 2004) and they have been shown to be important mediators of immunological tolerance (Manavalan et al. 2003; Kim-Schulze et al. 2006). They have been proposed to regulate the function of Toll-like receptors (TLR) and to alter the phenotype and profile of cytokine production by antigen presenting cells (Chang et al. 2002, 2009; Anderson and Allen 2009; Brown et al. 2009), thereby controlling both innate and adaptive immune responses. Their functions have been linked to control of bacterial and viral infection (Brown et al. 2009; Lee et al. 2007; Pilsbury et al. 2010). LILRs are genetically associated with autoimmune diseases such as multiple sclerosis (Koch et al. 2005; Ordóñez et al. 2009), Sjögren's syndrome (Kabalak et al. 2009), and rheumatoid arthritis (Kuroki et al. 2005; Huynh et al. 2007).

LILR receptors can be classified into two main groups, LILRA and LILRB. The LILRA group includes genes that have truncated cytoplasmic tails and associate with the $\gamma$ chain of FceRI through a charged arginine residue in the transmembrane domain, delivering an activating signal through an immunoreceptor tyrosine-based activation motif (ITAM) (Nakajima et al. 1999). One exception is LILRA3, a soluble protein that has no known signalling ability (Borges et al. 1997; Colonna et al. 1999). On the other hand, LILRB 
proteins have long cytoplasmic tails with immunoreceptor tyrosine-based inhibitory motifs (ITIM). Some LILR receptors interact with HLA class I (Group 1: LILRB1, -B2, -A1, $\mathrm{A} 2$ and $-\mathrm{A} 3$ ) and they share significant amino acid sequence homology over class I-binding regions (Borges et al. 1997; Colonna et al. 1998; Fanger et al. 1998; Willcox et al. 2003). The ligands for the other receptors, including LILRB3-B5 and LILRA5-A6 are unknown, except for LILRA4 which binds CD317 (Cao et al. 2009; Tavano et al. 2013). These receptors contain non-conservative substitutions in class I binding regions identified in group 1(Willcox et al. 2003).

LILR are encoded within the leukocyte receptor complex (LRC) on chromosome 19q13.4 (Fig. 1), adjacent to the related killer immunoglobulin (Ig)-like receptor (KIR) genes (Barrow and Trowsdale 2008). KIR genes exhibit considerable sequence polymorphism (Robinson et al. 2010) and extensive copy number variation (CNV) (Jiang et al. 2012). LILRB3 and LILRA6 are paired receptors which potentially deliver opposing signals. The genes encoding these receptors display remarkable diversity within their extracellular domains when compared to either LILRB1 or LILRB2 (Colonna et al. 1997). For example, there is evidence for variation in the number of copies of LILRA6 (Sudmant et al. 2010). In addition, it has been reported that some individuals lack LILRA3 gene expression as a consequence of a large deletion of $6.7 \mathrm{kbp}$, resulting in the removal of four Ig domain and two leader peptide exons from the genomic sequence (Torkar et al. 2000).

To gain insight into the genetics and functions of the polymorphic $L I L R$ that do not bind class I, we characterised variation in LILRB3 and LILRA6 genes and examined them for CNV.

\section{Materials and methods}

Samples

Sequencing analysis was performed in cDNA samples from 20 healthy individuals from the Cambridge Blood Centre. Informed consent was obtained from all individuals. CNV assay was carried out in DNA samples from 48 human cell lines from the International Histocompatibility Working Group from different ethnic origins (see Table S1).

\section{Sequencing}

RNA extraction and subsequent cDNA synthesis was performed as described previously (Jones et al. 2009). Transcripts were initially amplified from macrophage cDNA using primers NK1078 and NK1091 for LILRB3 (see Table S2 for primer details) and, NK1078 and NK636 for LILRA6. These initial polymerase chain reactions (PCRs) were performed using Phusion polymerase (Finnzymes) with the following cycling parameters: $30 \mathrm{~s}$ at $98{ }^{\circ} \mathrm{C}$ followed by 35 cycles of $98^{\circ} \mathrm{C}$ for $10 \mathrm{~s}, 68{ }^{\circ} \mathrm{C}$ for $30 \mathrm{~s}$, and $72{ }^{\circ} \mathrm{C}$ for $60 \mathrm{~s}$. All PCRs were performed on the MJ Research (Reno, NV, USA) PTC-200 thermal cycler. Products were assessed by cycle sequencing using BigDye Terminator version 3.1 methodology (Applied Biosystems) and an Applied Biosystems 3730xl DNA analyser using the primers NK1078 and NK1495 (LILRB3), or NK1078, NK1404 and NK1494 (LILRA6) (Table S2).

To determine the sequences of single alleles within heterozygote individuals, we performed PCRs on individual cDNAs, using forward primers with specificity for single nucleotide polymorphisms (SNPs) (Table S2). These SNPspecific primers (SSP) were used in conjunction with either the LILRB3 -specific reverse primer NK1091 or the LILRA6specific NK636. The cycling conditions were: $180 \mathrm{~s}$ at $96^{\circ} \mathrm{C}$, followed by 5 cycles of $96^{\circ} \mathrm{C}$ for $20 \mathrm{~s}, 70{ }^{\circ} \mathrm{C}$ for $45 \mathrm{~s}$, and $72{ }^{\circ} \mathrm{C}$ for $25 \mathrm{~s}$. This was followed by 31 cycles of $96^{\circ} \mathrm{C}$ for $25 \mathrm{~s}, 65^{\circ} \mathrm{C}$ for $50 \mathrm{~s}$ and $72{ }^{\circ} \mathrm{C}$ for $30 \mathrm{~s}$; followed by 4 cycles of $96^{\circ} \mathrm{C}$ for $30 \mathrm{~s}, 55^{\circ} \mathrm{C}$ for $60 \mathrm{~s}$ and $72^{\circ} \mathrm{C}$ for $90 \mathrm{~s}$. All amplicons from PCR-SSP carried an M13R tag at the $5^{\prime}$ end, which was exploited for subsequent cycle sequencing using the primers M13R, in addition to NK1495, NK1404 and NK1494.

Sequence analysis: calculation of $\mathrm{dN} / \mathrm{dS}$ ratios and statistical tests

To assess putative evolutionary selection pressure on polymorphism acting on the extracellular immunoglobulin-like region of LILRB3 and LILRA6 alleles, non-synonymous $(\mathrm{dN})$ and synonymous $(\mathrm{dS})$ substitution rates for all pairwise

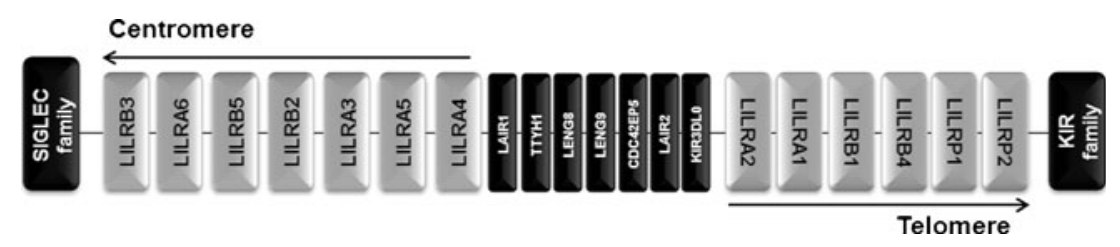

Fig. 1 LILR genes. The LILR family is located in the LRC on chromosome $19 q 13.4$ neighbouring the KIR family of genes. It is composed of two inverted clusters separated by LAIR and other genes. The transcription of each of these LILR clusters is in a head to tail fashion, as indicated by the arrows 
comparisons of alleles from both loci were calculated using the KaKs Calculator (Zhang et al. 2006), applying Nei and Gojobori's method and incorporating Jukes-Cantor correction (Nei and Gojobori 1986).

One-tailed Fisher's exact tests for positive selection for sequence pairs were performed on the entire extracellular Ig region of LILRB3 and LILRA6 using Graphpad (http://www. graphpad.com/quickcalcs/) as previously described (Zhang et al. 1997). Results were considered statistically significant with $p<0.05$.

\section{Quantitative PCR}

LILR copy number was determined by quantitative PCR (qPCR), on genomic DNA extracted from 48 human cell lines from the International Histocompatibility Working Group (http://www.ihwg.org/hla/) using the QIAamp DNA Blood Midi Kit (QIAGEN) following the manufacturer's instructions. The following genes were typed for CNV: LILRA1, LILRA2, LILRA3, LILRA4, LILRA5, LILRA6, LILRB1, LILRB2, LILRB3, LILRB4 and LILRB5.

Forward and reverse primers, and a dual-labelled probe were designed to specifically amplify each LILR gene (Table S3), avoiding any allelic variation identified to date. LILR sequences were analysed for specificity using the primerBLAST tool from the National Centre for Biotechnology Information (http://www.ncbi.nlm.nih.gov/ tools/primer-blast). In addition, all reactions contained specific primers and a probe for the STAT6 gene, which has two copies per diploid human genome, and was used as an endogenous reference gene. All reactions were performed in quadruplicate for each sample to increase the accuracy of copy number scoring.

A total of 10 ng of genomic DNA was amplified under the following PCR conditions: $5 \mathrm{~min}$ at $95{ }^{\circ} \mathrm{C}$; followed by 40 cycles of $95{ }^{\circ} \mathrm{C}$ for $15 \mathrm{~s}$ and $66^{\circ} \mathrm{C}$ for $50 \mathrm{~s}$; followed by 10 s at $40{ }^{\circ} \mathrm{C}$, using the LightCycler 480 System (Roche Diagnostics Ltd., Burgess Hill, UK). LILR copy number was determined by a quantitative PCR comparative Ct method (Schmittgen and Livak 2008).

\section{SSP-PCR genotyping}

Genotyping for presence of the SNP that encodes a threonine (T) at residue 94 within LILRA6 was performed on genomic DNA samples using the primer pair NK1513 (5'-CCCCCT GGAGCTGGTGAC-3') and NK559 (5'-TCATCAGAACAA AATGGTGATATCT-3') or NK560 (5'-CATCAGAACAAA ATGGTGATATCC-3'). Detection of a 570-bp deletion located within the intergenic region between LILRA6 and LILRB3 was performed using the primer pair NK1520 (5'-CTGGTC CCTGCAGTGGCA-3') and NK1518 (5'- GCCTTAGACT
TCCTATCCTGAAAC-3'). PCR cycling conditions were as previously described (Jones et al. 2006).

\section{Results}

LILRB3 and LILRA6 immunoglobulin-like domains are highly polymorphic

By exploiting polymorphic sites and unique regions within the $3^{\prime}$ ends of LILRB3 and LILRA6, we were able to characterise the sequences encoding the Ig domains of single alleles. This approach made it possible to identify individual allele sequences regardless of any variation in copy number (see later).

Analysis of the extracellular Ig domain-coding regions of the LILRB3 and LILRA6 genes, using cDNA samples from 20 healthy individuals, identified substantial variation in the nucleotide sequences of both receptors (Tables 1 and 2). This analysis generated 25 different sequences, 21 of which were novel and have subsequently been deposited in Genbank with accession numbers from KF294233 to KF294253.

We elucidated a total of 41 variable nucleotides in LILRB3 and LILRA6 sequences. Twenty-one of the non-synonymous and three of the synonymous substitutions affected the same nucleotide position in the coding sequence of both genes. None of these polymorphisms displayed significant linkage disequilibrium between LILRB3 and LILRA6 alleles (data not shown). The inhibitory receptor LILRB3 encompassed 40 polymorphic sites with 34 non-synonymous and six synonymous substitutions. The activating receptor LILRA6 showed less variation; 29 polymorphic sites, of which 25 were nonsynonymous and four synonymous substitutions (Tables 1 and 2). Moreover, the analysis of these cDNA sequences revealed that four individuals carried three alleles of LILRA6, of which LILRA6*01 and *03 were common to all the samples and in three out of the four samples, the third allele was LILRA6*04. LILRA6*03 was present in the cDNA samples of all individuals with three alleles and it was the only LILRA6 allele with the SNP $94 \mathrm{~T}$ (Table 1), suggesting that this SNP correlated with the increase in the number of copies of LILRA6. We performed SSP-PCR to detect the polymorphism at position 94 in the genomic DNA samples from cell lines but did not find any association between the presence of $\mathrm{T}$ at position 94 and the increase in the number of copies of LILRA6 in our cohort (Table 3).

Protein evolution by selective pressure can be measured by considering the replacement of nucleotides within codons and by analysing site-by-site the $\mathrm{dN} / \mathrm{dS}$ substitution ratio between sequences (Yang et al. 2000). This analysis can reveal the presence of either strictly conserved $(\mathrm{dN} / \mathrm{dS}<1)$ or rapidly 


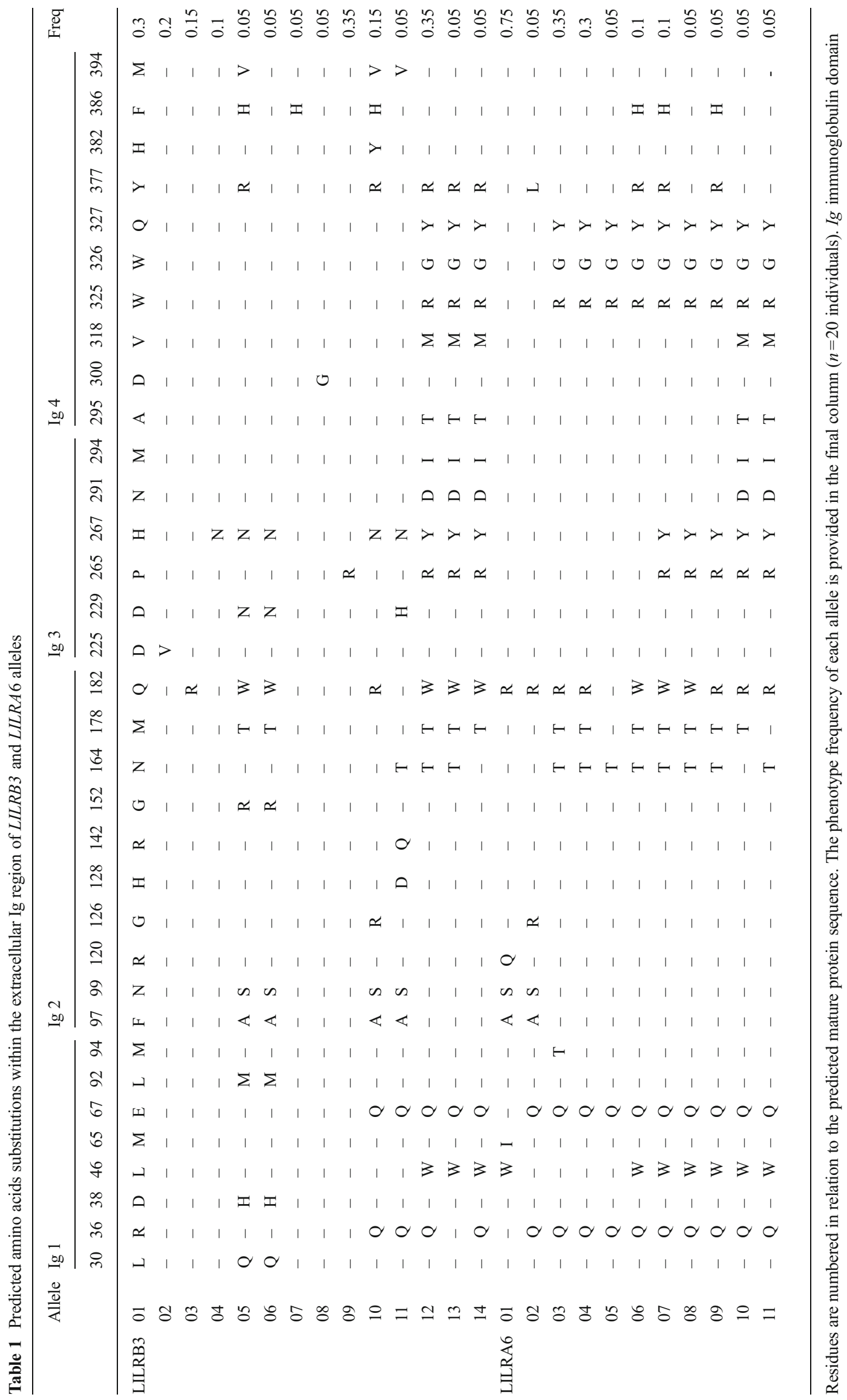


Table 2 Synonymous polymorphism within the extracellular Ig-coding region of LILRB3 and LILRA6 alleles

\begin{tabular}{|c|c|c|c|c|c|c|c|c|}
\hline & \multirow[b]{2}{*}{ Allele } & \multirow{2}{*}{$\begin{array}{l}\text { Ig } 1 \\
70(\mathrm{~A}) \\
\text { GCG }\end{array}$} & \multirow{2}{*}{$\begin{array}{l}\operatorname{Ig} 2 \\
\text { 98(Y) } \\
\text { TAㅁ }\end{array}$} & \multicolumn{3}{|l|}{ Ig 3} & \multicolumn{2}{|l|}{$\operatorname{Ig} 4$} \\
\hline & & & & $\begin{array}{l}267(\mathrm{H} / \mathrm{N} / \mathrm{Y})^{\mathrm{a}} \\
\mathrm{H}: \mathrm{CAC}\end{array}$ & $\begin{array}{l}\text { 274(Y) } \\
\text { TAT }\end{array}$ & $\begin{array}{l}287(\mathrm{~S}) \\
\mathrm{AGC}\end{array}$ & $\begin{array}{l}326(\mathrm{G})^{\mathrm{a}} \\
\text { G: GGG }\end{array}$ & $\begin{array}{l}392(E) \\
\text { GAA }\end{array}$ \\
\hline \multirow[t]{14}{*}{ LILRB3 } & 01 & -- & -- & H: - - - & -- &.- & W &.- \\
\hline & 01 & -- & -- & H: - - - & - - &.- & W & $-\ldots$ \\
\hline & 03 & -- & -- & H: - - - & -- & -- & W & -- \\
\hline & 04 & -- & -- & $\mathrm{N}: \mathrm{A}-\mathrm{T}$ & -- & -- & W & $-\ldots$ \\
\hline & 05 & $--\mathrm{A}$ & -- & $\mathrm{N}: \mathrm{A}-\mathrm{T}$ & $--\mathrm{C}$ & -- & W & - - G \\
\hline & 06 & $--\mathrm{A}$ & -- & $\mathrm{N}: \mathrm{A}-\mathrm{T}$ & $--\mathrm{C}$ & - - - & W & -- \\
\hline & 07 & --- & -- & H: - - - & -- & - - & W & - - \\
\hline & 08 & --- & -- & H: - - - & --- & -- & W & -- \\
\hline & 09 & --- & -- & H: - - - & -- & -- & W & -- \\
\hline & 10 & $--\mathrm{A}$ & $--\mathrm{T}$ & $\mathrm{N}: \mathrm{A}-\mathrm{T}$ & $--\mathrm{C}$ &.- & W & $--G$ \\
\hline & 11 & $--\mathrm{A}$ & -- & $\mathrm{N}: \mathrm{A}-\mathrm{T}$ & -- &.- & W & $--G$ \\
\hline & 12 & $--\mathrm{A}$ & -- & Y: T - - & -- & $--\mathrm{T}$ & G: - - - & -- \\
\hline & 13 & $--\mathrm{A}$ & -- & Y: T - - & -- & $--\mathrm{T}$ & G: - - - & -- \\
\hline & 14 & $--\mathrm{A}$ & -- & Y: T - - & - - & $--\mathrm{T}$ & G: - - - & -- \\
\hline \multirow[t]{11}{*}{ LILRA6 } & 01 & -- & $--\mathrm{T}$ & H: - - - & -- & -- & W & -- \\
\hline & 02 & $--\mathrm{A}$ & $--\mathrm{T}$ & H: - - - & -- & - - - & W & - - - \\
\hline & 03 & $--\mathrm{A}$ & -- & H: - - - & -- & --- & G: - - T & -- \\
\hline & 04 & $--\mathrm{A}$ & -- & H: - - - & -- & - - - & G: - - T & -- \\
\hline & 05 & $--\mathrm{A}$ & -- & H: - - - & -- & -- & G: - - T & -- \\
\hline & 06 & $--\mathrm{A}$ & -- & H: - - - & -- & -- & G: - - T & - - G \\
\hline & 07 & $--\mathrm{A}$ & -- & Y: T - - & -- & -- & G: - - T & $--\mathrm{G}$ \\
\hline & 08 & $--\mathrm{A}$ & -- & Y: T - - & --- & --- & G: - - T & -- \\
\hline & 09 & $--\mathrm{A}$ & --- & Y: T - - & --- & -- & G: - - T & - - G \\
\hline & 10 & $--\mathrm{A}$ & -- & Y: T - - & --- & -- & G: - - - & -- \\
\hline & 11 & $--\mathrm{A}$ & -- & Y: T - - & -- & - - - & G: - - - & -- \\
\hline
\end{tabular}

Residue number, amino acid and codon sequences are displayed (residues are numbered in relation to the predicted mature protein sequence). In all cases, synonymous polymorphism occurs at the third position within each codon (underlined)

${ }^{a}$ Sites where both synonymous and non-synonymous polymorphisms occur in conjunction

evolving regions in genes ( $\mathrm{dN} / \mathrm{dS}>1)$. LILRB3 and LILRA6 sequences displayed in Tables 1 and 2 were analysed in this manner. There were predominantly non-synonymous polymorphisms, so the $\mathrm{dN} / \mathrm{dS}$ ratios were consistent with positive selection (Fig. 2).

LILRA3 and LILRA6 genes, but not LILRB3, show variation in copy number

We designed assays to detect variation in the number of copies of LILR genes by qPCR. CNV was detected in the LILRA3 and LILRA6 loci (Fig. 3c and f, respectively). LILRA3 varied between zero, one or two copies per diploid genome. Around $21 \%$ of the samples lacked LILRA3, while $\sim 19 \%$ had only one copy of this gene. CNV in LILRA6 varied between one, two, three and four copies per diploid genome; $\sim 8 \%$ of the samples had a single copy of the gene, whilst $\sim 33 \%$ carried a duplication of LILRA6 (three or four copies). Despite its high rate of allelic variation, LILRB3 had a constant number of two copies per genotype (Fig. 4c).

We identified a fosmid sequence deposited in Genbank (accession number: AC236241) that contains two sequences of LILRA6, the arrangement of which is represented in Fig. 5. The additional LILRA6 locus (LILRA6 [2]) occurred $\sim 15 \mathrm{~kb}$ upstream of the common LILRA6 locus (LILRA6 [1]) within the region normally occupied by LILRB3. Sequence AC236241 also possesses a deletion within a tandem repeat sequence that is normally located within the intergenic region between LILRA6 and LILRB3. We used the Tandem repeats finder program (Benson 1999) to detect the 33-bp tandem repeat sequence, which has a consensus sequence of TCTA TTGAGATCCTATGGAGGTCCTGTGGGGGT and a copy 
Table 3 LILRA6 CNV and presence of tandem repeat sequence and SNP $94 \mathrm{~T}$
$C N$ calculated copy number, $T R S$ tandem repeat sequence

\begin{tabular}{|c|c|c|c|c|c|c|c|}
\hline Cell line & $\mathrm{CN}$ & TRS & $94 \mathrm{~T}$ & Cell line & $\mathrm{CN}$ & TRS & $94 \mathrm{~T}$ \\
\hline KT12 & 2.89 & Deletion & Negative & $\mathrm{RSH}$ & 3.32 & Deletion & Negative \\
\hline TAB089 & 2.06 & Complete & Negative & $\mathrm{TL}$ & 3.04 & Deletion & Positive \\
\hline XL1-ND & 2.31 & Complete & Negative & PAR & 2.06 & Complete & Negative \\
\hline RML & 3.02 & Deletion & Negative & WT24 & 3.19 & Deletion & Positive \\
\hline KY & 1.68 & Complete & Negative & SA & 2.10 & Complete & Positive \\
\hline HOM2 & 1.76 & Complete & Negative & LATIF & 1.86 & Complete & Negative \\
\hline KT17 & 2.01 & Deletion & Negative & OZB & 1.05 & Complete & Negative \\
\hline JESTHOM & 1.98 & Complete & Negative & AMAI & 2.84 & Complete & Positive \\
\hline AKIBA & 1.92 & Complete & Negative & $\mathrm{COX}$ & 1.96 & Complete & Positive \\
\hline WWPAK & 1.90 & Complete & Negative & KAS011 & 0.38 & Complete & Negative \\
\hline GRC & 3.28 & Deletion & Negative & $\mathrm{CO} 47$ & 2.11 & Deletion & Positive \\
\hline BM9 & 2.27 & Complete & Negative & FPAF & 1.97 & Deletion & Negative \\
\hline SLE005 & 2.10 & Deletion & Positive & SNA BLL & 2.01 & Complete & Negative \\
\hline DUCAF & 3.09 & Deletion & Negative & DUGE & 2.98 & Deletion & Negative \\
\hline PMH 161 & 2.00 & Complete & Negative & WJR076 & 1.93 & Complete & Negative \\
\hline LB & 1.95 & Complete & Negative & VAVY & 2.01 & Complete & Negative \\
\hline BTB & 1.96 & Deletion & Negative & HOR & 1.10 & Complete & Negative \\
\hline IBW9 & 3.09 & Deletion & Positive & MANIKA & 3.89 & Deletion & Positive \\
\hline DKB & 3.13 & Deletion & Positive & MGAR & 2.32 & Deletion & Negative \\
\hline DEM & 3.08 & Deletion & Positive & H0104 & 1.84 & Deletion & Negative \\
\hline $\mathrm{LBF}$ & 2.90 & Deletion & Positive & M7 & 2.70 & Deletion & Negative \\
\hline BOLETH & 1.16 & Complete & Negative & LCL 721 & 2.13 & Complete & Positive \\
\hline HO301 & 2.01 & Deletion & Positive & CRGE & 2.04 & Complete & Negative \\
\hline 31708 & 3.75 & Deletion & Negative & $\mathrm{MCF}$ & 1.99 & Deletion & Positive \\
\hline
\end{tabular}

number of 19.9. This repeat was absent from AC236241. By utilising PCR primers that flank this deletion (Fig. 5b), we found that its presence correlated strongly with the duplication of LILRA6 within our cohort, occurring in around $93 \%$ of the samples with more than two copies of LILRA6 (Table 3), indicating that fosmid AC236241 represents a commonly occurring haplotype that carries a duplication of LILRA6.

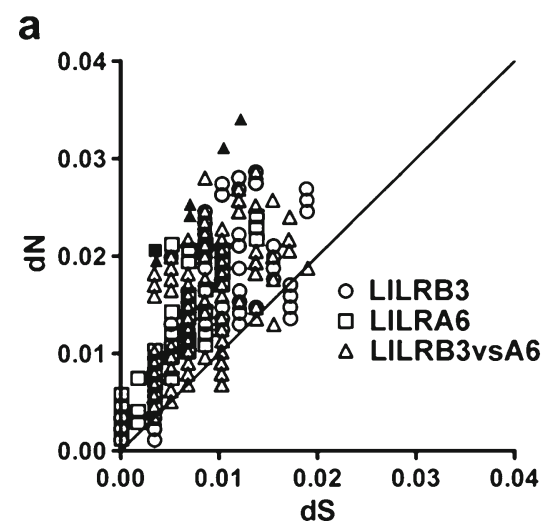

Fig. $2 \mathrm{dN} / \mathrm{dS}$ ratios of the extracellular regions of LILRB3 and LILRA6 indicate positive selection. a $\mathrm{dN}$ and $\mathrm{dS}$ values from allelic pairwise comparisons of each gene, LILRB3 (circles) and LILRA6 (squares), and inter-gene comparison (triangles). Only sequences detected in this study were included in the analysis. The central plot line

\section{No other LILR gene shows CNV}

To determine whether the CNV we observed was a more widespread feature of LILR genes, we carried out CNV analysis of all other activating and inhibitory LILR genes (LILRA1, $-A 2,-A 4,-A 5,-B 1,-B 2,-B 4$ and $-B 5$ ). This analysis did not identify any further variation in the number

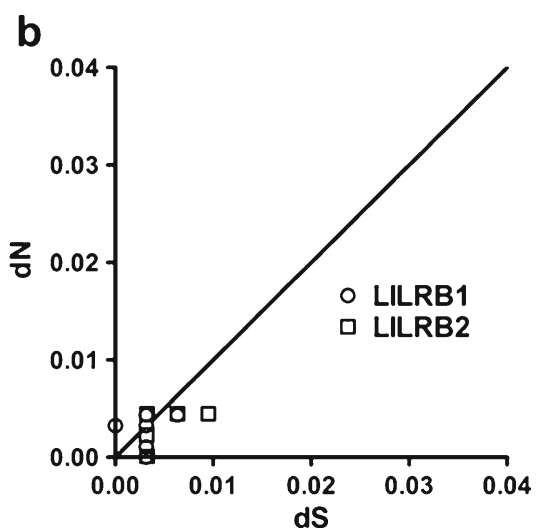

represents balancing selection (where $\mathrm{dN}=\mathrm{dS}$ ). Shaded symbols indicate significance using a Fisher's exact test for positive selection pressure (Zhang et al. 1997). b $\mathrm{dN}$ and $\mathrm{dS}$ values for previously reported LILRB1 (circles) and LILRB2 (squares) sequences deposited in Genbank (http://www.ncbi.nlm.nih.gov/genbank/) 
Fig. 3 Copy number variation of activating LILR receptor genes.

Summaries of the results of copy number assays for LILRA1 (a), LILRA2 (b), LILRA3 (c), LILRA4 (d), LILRA5 (e) and LILRA6 (f). The number of copies per genome was constant (two copies) in activating LILR receptors with the exception of LILRA3 and LILRA6, which displayed variation. LILRA3 showed from zero to two copies per genome. LILRA6 showed from one to four copies per genome. Each bar represents the calculated copy number obtained for one sample (see Table S1).

Number inside plot is the predicted copy number for samples

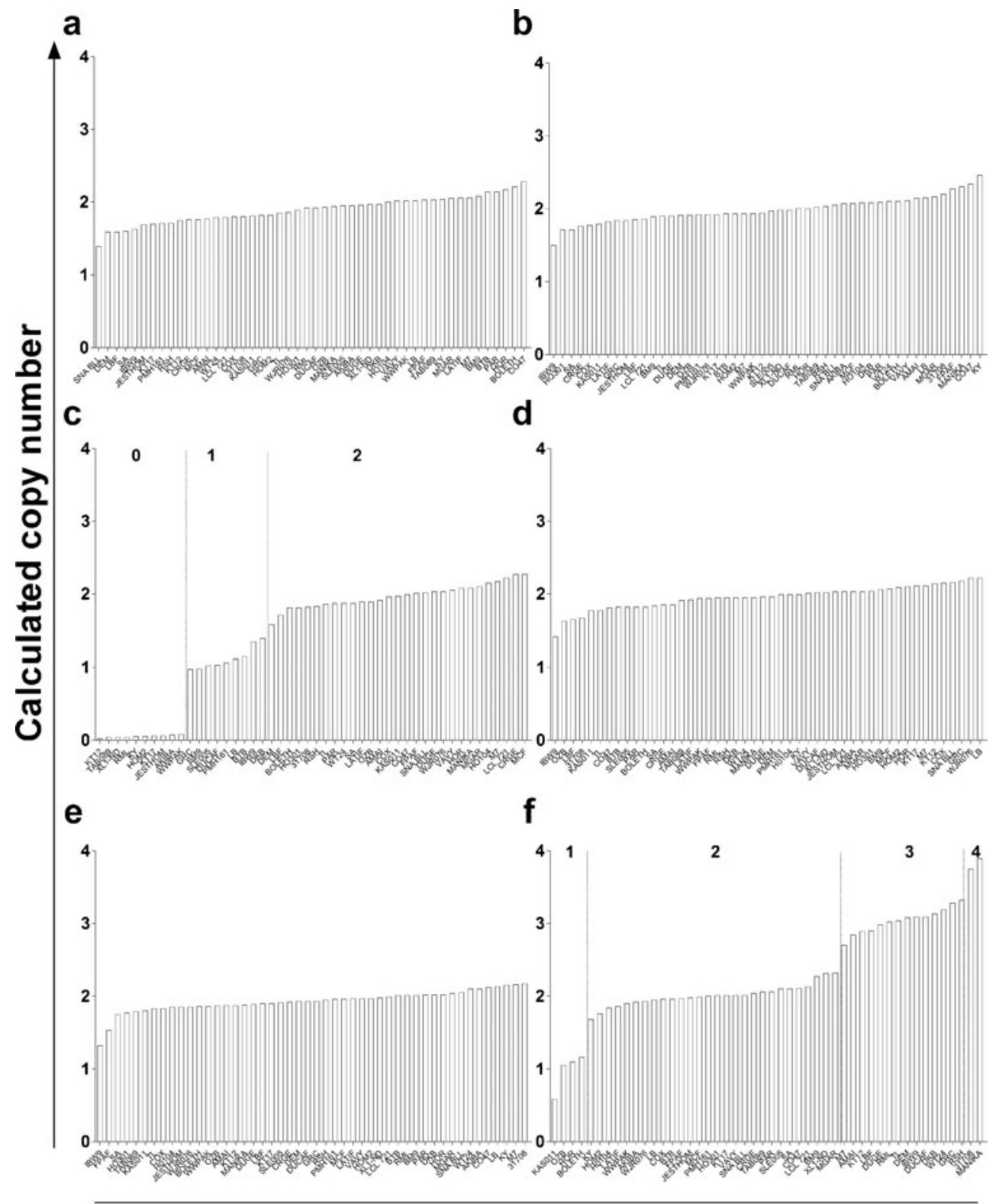

Samples

of copies of other LILR genes (Figs. 3 and 4), indicating that CNV is specific to the LILRA3 and LILRA6 loci, or rare for other genes.

\section{Discussion}

Amplification and sequencing of LILRB3 and LILRA6 cDNA from 20 individuals returned 25 different sequences with 41 polymorphic sites, consistent with these receptors being highly polymorphic in their extracellular domains (Colonna et al. 1997). Most of variable positions were represented in both LILRB 3 and LILRA6, including several synonymous substitutions. This indicates the possibility of genetic transfer between these two genes and the additional copies of LILRA6. The patchwork nature of the variation is reminiscent of the variation in some MHC class I or class II genes, in which case it may be achieved by allele or gene conversion (Traherne et al. 2006). Several non-synonymous polymorphisms within LILRB3 and LILRA6 occur at positions known to form part of the binding sites of Group 1 LILR to MHC class I: 36, 38, 67, 97, 99 and 126 (Willcox et al. 2003; Yang and Bjorkman 2008). Although no specific ligand for both LILRB3 and LILRA6 receptors has yet been identified, it is tempting to speculate that these variations may have functional consequences. Additionally, we found three alleles of LILRA6 in 

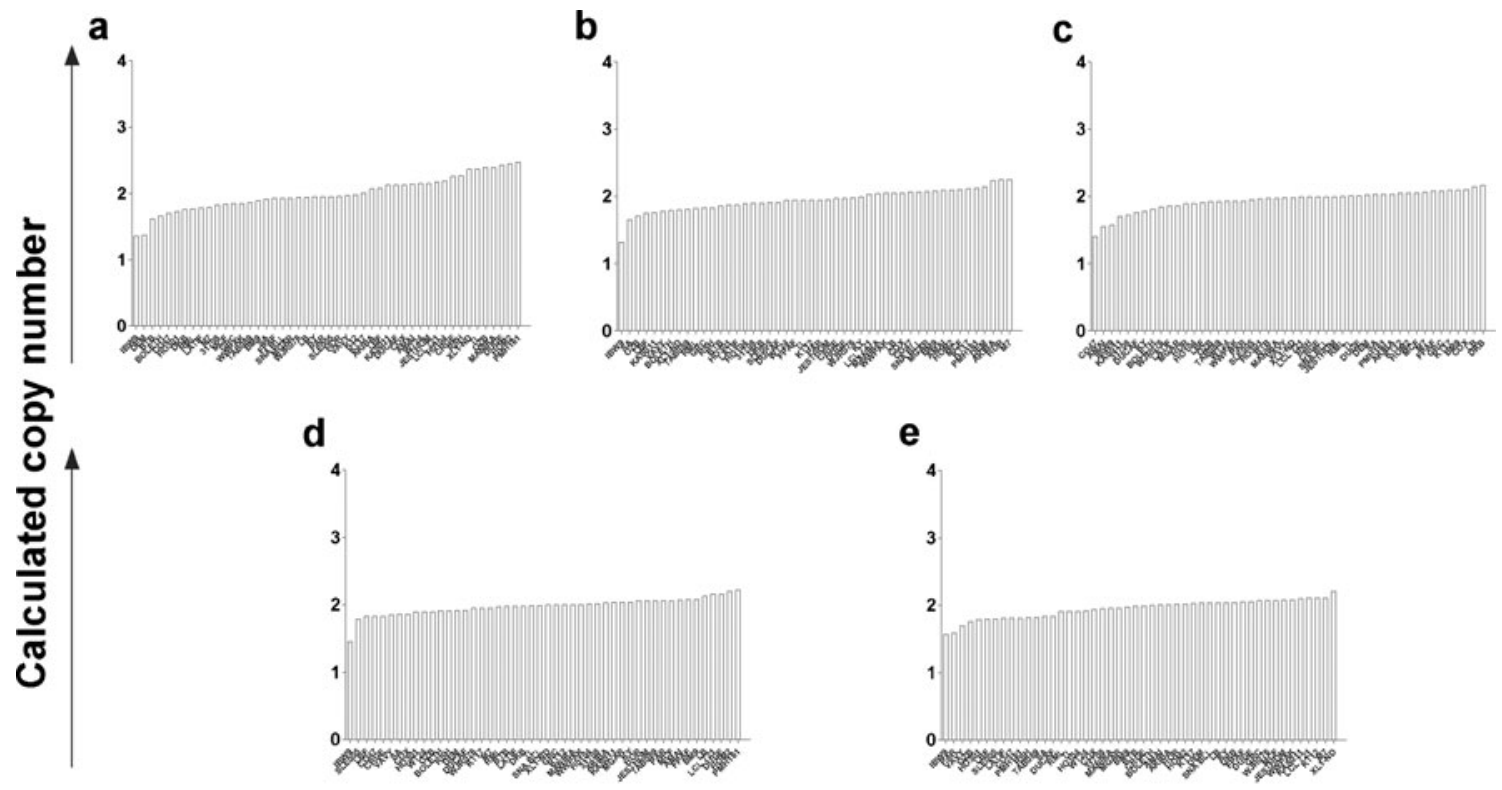

\section{Samples}

Fig. 4 Copy number variation of inhibitory LILR receptor genes. Copy number variation was analysed for the inhibitory receptor genes $L I L R B I$ (a), LILRB2 (b), LILRB3 (c), LILRB4 (d) and LILRB5 (e). The figure

four cDNA samples, indicating that the duplicated copies of this gene are expressed and are presumably functional. The fact that three out of four samples had the combination of LILRA6*03 and LILRA6*04 concurs with the duplication of LILRA6 found in a fosmid (accession number AC236241). However, this allele pairing was not found in all tri-allelic individuals. Moreover, the SNP $94 \mathrm{~T}$, characteristic of LILRA6*03, which was carried by all four individuals with shows the results of this analysis where each of these receptor genes are present in two copies per genotype. Each bar represents the calculated copy number obtained for one sample (see Table S1)

three alleles, was not associated with the increase in the number of copies of LILRA6 found in cell lines. Therefore, it would appear that the allele content within the haplotype comprising duplicated LILRA6 genes is variable.

The method of $\mathrm{CNV}$ analysis we describe has a number of advantages: it is simple to carry out, low cost, specific and sensitive and is high-throughput, as up to a thousand samples can be analysed per week. Variation in LILR gene copy

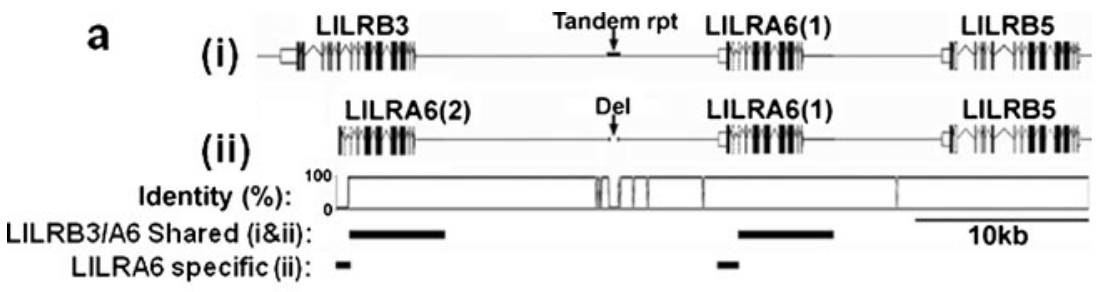

b

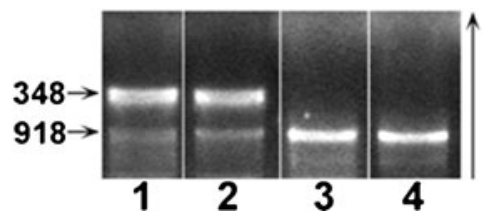

Fig. 5 Genetic organisation of the centromeric region of the LILR gene cluster encompassing LILRB5, LILRB3 and LILRA6. a Genes are arranged according to their positions as determined by analysis of the representative sequence CU151838 (i) and within fosmid AC236241 (ii). Sequence AC236241 carries two copies of LILRA6 (labelled 1 and 2) and a 570-bp deletion within the tandem repeat region present in CU151838. The comparative homology between these two sequences is also presented. Regions occurring within both LILRB3 and LILRA6 that share $>95 \%$ identity are indicated, together with the locations of LILRA6-

specific sequence within AC236241. The AC236241 sequence terminates within the 3' region of LILRA6 (2). b PCR-based detection of the deleted region identified within fosmid AC236241. Amplification of the tandem repeat sequence between LILRB3 and LILRA6 (918 bp) or two LILRA6 sequences (348 bp). Samples are DEM (1), DUCAF (2), HOR (3), and SA (4). The arrow indicates the direction of DNA migration. Refer to Table 3 for information about the results of LILRA6 copy number, the deletion within the tandem repeat sequence and results for the SNP 94 T by SSP-PCR 
number was detected within LILRA3, which encodes a soluble molecule (Borges et al. 1997; Colonna et al. 1999), and the activating LILRA6, while all inhibitory LILR genes, including the highly polymorphic $L I L R B 3$, had a constant number of two copies per diploid genome. The gene frequency of deletion of LILRA3 was around $40 \%$, in concordance with previously published results where the frequency of deletions varied from $6 \%$ to $84 \%$ in different populations (Torkar et al. 2000; Wiśniewski et al. 2013). LILRA6 was more variable; $8.3 \%$ of the samples showed a heterozygous deletion of LILRA6, and in $33.3 \%$ of the cases, we observed duplications of this gene (three or four copies).

The variation in the number of copies of LILRA6 could be due to non-allelic homologous recombination (NAHR) or crossing-over between sequences that are not in allelic positions, a phenomenon proposed for CNV in KIR (Traherne et al. 2010). These events are mediated by genomic structures that are flanked by paralogous repeat sequences, low-copy repeats, segmental duplications or tandem repeat regions (Jeffreys et al. 2004; Liu et al. 2012). We found a tandem repeat sequence between LILRB3 and LILRA6 genes (Fig. 5a), that was deleted in sequence contained in fosmid AC236241, which carries a duplication of LILRA6. Therefore, it could be hypothesised the deletion within the tandem repeat region may be mechanistically linked to the duplication event of LILRA6 within this haplotype. From analysis of the AC236241 sequence an obvious insertion point of the duplicated LILRA6 gene could not be identified indicating that unequal crossover could have occurred within the $\sim 4.9-\mathrm{kb}$ homologous region that LILRA6 shares with LILRB3 (Fig. 5a). Recombination centred upon the repeat region may account for the lack of significant linkage disequilibrium observed between alleles of LILRB3 and LILRA6, as determined by the analysis of cDNA from 20 individuals (data not shown).

Our CNV screen indicates that LILRA6 is absent on some haplotypes. There are two sequences in Genbank (accession numbers: AC235034 and AC153469) that lack LILRA6, and instead carry LILRB3 at that genomic location. The LILRB3 allele within AC235034 encodes a predicted protein sequence that is identical to LILRA6*01 throughout its extracellular region, while the predicted protein encoded by $\mathrm{AC} 153469$ only differs with $L I L R A 6^{*} 01$ due to the presence of $164 \mathrm{~T}$. LILRA6*01 is the most frequently occurring allele according to our cDNA screen (Table 1) and carries two unique SNPs (65 I and $120 \mathrm{Q}$ ), and a unique combination of variable positions $36 \mathrm{R}, 46 \mathrm{~W}$ and $67 \mathrm{E}$. We did not detect these polymorphic features within any LILRB3 allele, suggesting that they are predominately a characteristic of LILRA6. Their presence within the LILRB3 sequences of AC153469 and AC235034 is evidence of an unequal crossover event that has occurred within the homologous region following the Ig2-encoding exon of LILRA6, resulting in the replacement of LILRA6- specific sequence within the $3^{\prime}$ of the gene with that of LILRB3. This indicates that unequal crossover is a mechanism by which genetic information may be exchanged between these two genes (Fig. 6a). An additional possibility could be the formation of single-stranded DNA (ssDNA) secondary structures or hairpin loops in the repeat tandem sequence (Fig. 6b). These structures are accessible substrates for nucleases, making them susceptible to strand breaks. This may lead to repair processes that can cause deletion of regions or genetic transfer of homologous sequences between chromosomes. Such recombination processes have previously been proposed in the evolution of the KIR gene cluster (Traherne et al. 2010).

Functionally, the increase in the number of copies of LILRA6 could affect the balance between activating and inhibitory signals after ligand binding. How could the combination of CNV and variable sequences be interpreted in terms of function? So far, it is difficult to identify haplotype-specific combinations of variable sequences over the sets of genes, as we do not have sequence information over single haplotypes. If these exist they would be consistent with haplotype-specific interactions with a ligand and integration of signals from the different receptors, as may be the case for some neighbouring KIR A and B haplotypes. They would suggest that the
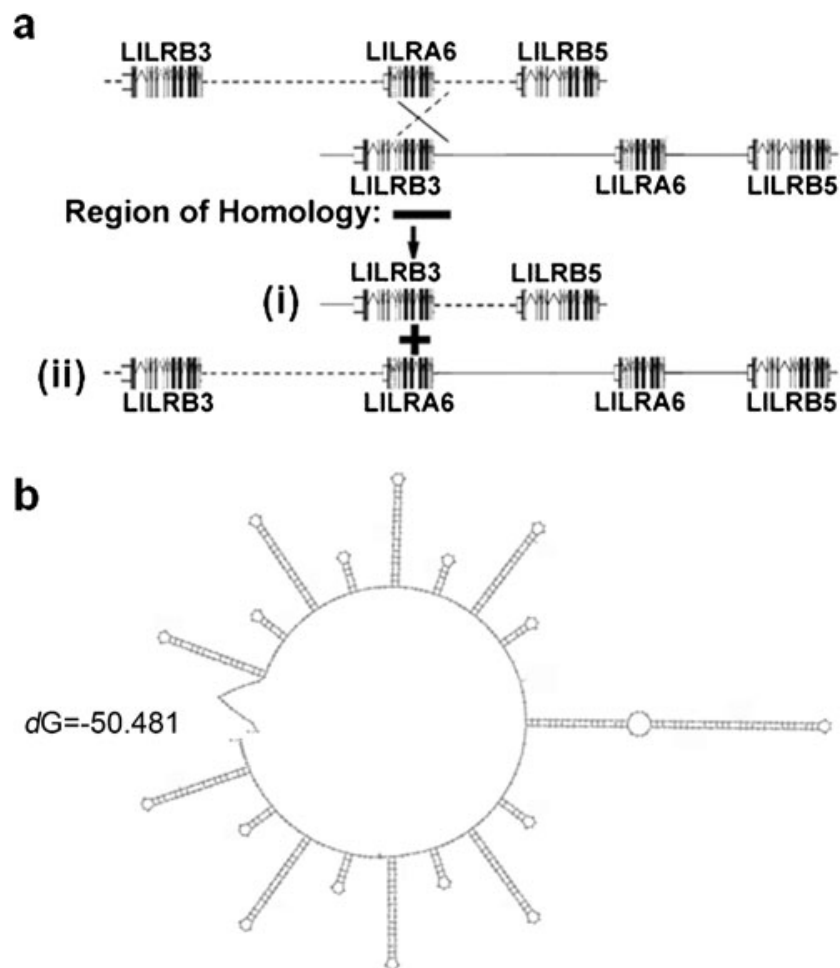

Fig. 6 Non-allelic homologous recombination and potential ssDNA secondary structures produced at the tandem repeat sequence. a The process of unequal crossing-over of homologous sequences proposed to generate deletions (i) or duplications (ii) of LILRA6. b Seventeen hairpin structures were predicted using the Mfold program (Zuker 2003). Twenty copies of the repeat tandem sequence TCTATTGAGATCCTATGGAG GTCCTGTGGGGGT was used as the input 
constellations of variable activating and inhibitory receptors on haplotypes are driven by interaction with a variable ligand, such as a variable pathogen.

In conclusion, we have shown high levels of polymorphism and homology between LILRB3 and LILRA6 in their extracellular domains, with LILRA6 also displaying alterations in the number of copies. All the variations observed in these genes may affect ligand binding, the equilibrium between activating and inhibitory signals, and therefore, the balance of immune response. The allelic variation and $\mathrm{CNV}$ in the LILRB3/LILRA6 pair we have described is consistent with strong selection for variation and high $\mathrm{dN} / \mathrm{dS}$ ratios are characteristic of other immune system genes, such as MHC or KIR, that are involved with resistance to pathogens (Park et al. 2012).

Acknowledgements This work was funded by the Medical Research Council (MRC) and Association for International Cancer Research (AICR) with additional support from the Wellcome Trust and the National Institute for Health Research (NIHR) Cambridge Biomedical Research Centre. María Rocío López-Álvarez was funded by Ministerio de Educación of Spain, under the program "Programa Nacional de Movilidad de Recursos Humanos del Plan Nacional de I-D+i 2008-2011" and Fundación Séneca 04087/GERM/06 Project.

Conflict of interest The authors declare they have no conflict of interest.

Open Access This article is distributed under the terms of the Creative Commons Attribution License which permits any use, distribution, and reproduction in any medium, provided the original author(s) and the source are credited.

\section{References}

Anderson KJ, Allen RL (2009) Regulation of T-cell immunity by leucocyte immunoglobulin-like receptors: innate immune receptors for self on antigen-presenting cells. Immunology 127:8-17

Barrow AD, Trowsdale J (2008) The extended human leukocyte receptor complex: diverse ways of modulating immune responses. Immunol Rev 224:98-123

Benson G (1999) Tandem repeats finder: a program to analyze DNA sequences. Nucleic Acid Res 27(2):573-580

Borges L, Hsu ML, Fanger N, Kubin M, Cosman D (1997) A family of human lymphoid and myeloid Ig-like receptors, some of which bind to MHC class I molecules. J Immunol 159:5192-5196

Brown D, Trowsdale J, Allen R (2004) The LILR family: modulators of innate and adaptive immune pathways in health and disease. Tissue Antigens 64(3):215-225

Brown DP, Jones DC, Anderson KJ et al (2009) The inhibitory receptor LILRB4 (ILT3) modulates antigen presenting cell phenotype and, along with LILRB2 (ILT4), is upregulated in response to Salmonella infection. BMC Immunol 10:56

Cao W, Bover L, Cho M et al (2009) Regulation of TLR7/9 responses in plasmacytoid dendritic cells by BST2 and ILT7 receptor interaction. J Exp Med 206(7):1603-1614

Chang CC, Ciubotariu R, Manavalan JS et al (2002) Tolerization of dendritic cells by $\mathrm{T}(\mathrm{S})$ cells: the crucial role of inhibitory receptors ILT3 and ILT4. Nat Immunol 3(3):237-243
Chang CC, Liu Z, Vlad G et al (2009) Ig-like transcript 3 regulates expression of proinflammatory cytokines and migration of activated T cells. J Immunol 182:5208-5216

Colonna M, Navarro F, Bellon T et al (1997) A common inhibitory receptor for major histocompatibility complex class I molecules on human lymphoid and myelomonocytic cells. J Exp Med 186:18091818

Colonna M, Samaridis J, Cella M et al (1998) Human myelomonocytic cells express an inhibitory receptor for classical and nonclassical MHC class I molecules. J Immunol 160:3096-3100

Colonna M, Nakajima H, Navarro F, Lopez-Botet M (1999) A novel family of Ig-like receptors for HLA class I molecules that modulate function of lymphoid and myeloid cells. J Leukoc Biol 66:375-381

Fanger NA, Cosman D, Peterson L, Braddy SC, Maliszewski CR, Borges L (1998) The MHC class I binding proteins LIR-1 and LIR-2 inhibit Fc receptor-mediated signaling in monocytes. Eur J Immunol 28:34233434

Huynh OA, Hampartzoumian T, Arm JP et al (2007) Down-regulation of leucocyte immunoglobulin-like receptor expression in the synovium of rheumatoid arthritis patients after treatment with disease-modifying antirheumatic drugs. Rheumatology (Oxford) 46(5):742-751

Jeffreys AJ, Holloway JK, Kauppi L et al (2004) Meiotic recombination hot spots and human DNA diversity. Philos Trab R Soc London B Biol 59(1441):141-152

Jiang W, Johnson C, Jayaraman J et al (2012) Copy number variation leads to considerable diversity for B but not A haplotypes of the human KIR genes encoding NK cell receptors. Genome Res 22(10): $1845-1854$

Jones DC, Edgar RS, Ahmad T et al (2006) Killer Ig-like receptor (KIR) genotype and HLA ligand combinations in ulcerative colitis susceptibility. Genes Immun 7(7):576-582

Jones DC, Roghanian A, Brown DP et al (2009) Alternative mRNA splicing creates transcripts encoding soluble proteins from most LILR genes. Eur J Immunol 39(11):3195-3206

Kabalak G, Dobberstein SB, Matthias T, Reuter S, The YH, Dörner T, Schmidt RE, Witte T (2009) Association of immunoglobulin-like transcript 6 deficiency with Sjögren's syndrome. Arthritis Rheum 60(10):2923-2925

Kim-Schulze S, Scotto L, Vlad G et al (2006) Recombinant Ig-like transcript $3-\mathrm{Fc}$ modulates $\mathrm{T}$ cell responses via induction of Th energy and differentiation of CD8 ${ }^{+}$T suppressor cells. J Immunol 176(5):2790-2798

Koch S, Goedde R, Nigmatova V et al (2005) Association of multiple sclerosis with ILT6 deficiency. Genes Immun 6:445-447

Kuroki K, Tsuchiya N, Shiroishi M et al (2005) Extensive polymorphisms of LILRB1 (ILT2, LIR1) and their association with HLADRB1 shared epitope negative rheumatoid arthritis. Hum Mol Genet 14(16):2469-2480

Lee DJ, Sieling PA, Ochoa MT et al (2007) LILRA2 activation inhibits dendritic cell differentiation and antigen presentation to T cells. J Immunol 179(12):8128-8136

Liu P, Carvalho CM, Hastings PJ, Lupski JR (2012) Mechanisms for recurrent and complex human genomic rearrangements. Curr Opin Genet Dev 22(3):211-220

Manavalan JS, Rossi PC, Vlad G et al (2003) High expression of ILT3 and ILT4 is a general feature of tolerogenic dendritic cells. Transpl Immunol 11(3-4):245-258

Nakajima H, Samaridis J, Angman L, Colonna M (1999) Human myeloid cells express an activating ILT receptor (ILT1) that associates with Fc receptor gamma-chain. J Immunol 162(1):5-8

Nei M, Gojobori T (1986) Simple methods for estimating the numbers of synonymous and nonsynonymous nucleotide substitutions. Mol Biol Evol 3(5):418-426

Ordóñez D, Sánchez AJ, Martínez-Rodríguez JE et al (2009) Multiple sclerosis associates with LILRA3 deletion in Spanish patients. Genes Immun 10(6):579-585 
Park S, Yang JS, Kim J et al (2012) Evolutionary history of human disease genes reveals phenotypic connections and comorbidity among genetic diseases. Sci Rep 2:757

Pilsbury LE, Allen RL, Vordermeier M (2010) Modulation of Toll-like receptor activity by leukocyte Ig-like receptors and their effects during bacterial infection. Mediat Inflamm 2010:536478

Robinson J, Mistry K, McWilliam H, Lopez R, Marsh SG (2010) IPD the Immuno Polymorphism Database. Nucleic Acids Res 38:D863D869

Schmittgen TD, Livak KJ (2008) Analyzing real-time PCR data by the comparative C(T) method. Nat Protoc 3:1101-1108

Sudmant PH, Kitzman JO, Antonacci F et al (2010) Diversity of human copy number variation and multicopy genes. Science 330(6004): 641-646

Tavano B, Galao RP, Graham DR et al (2013) Ig-like transcript 7, but not bone marrow stromal cell antigen 2 (also known as HM1.24, tetherin, or CD317), modulates plasmacytoid dendritic cell function in primary human blood leukocytes. J Immunol 190(6):2622-2630

Torkar M, Haude A, Milne S, Beck S, Trowsdale J, Wilson MJ (2000) Arrangement of the ILT gene cluster: a common null allele of the ILT6 gene results from a $6.7-\mathrm{kbp}$ deletion. Eur J Immunol 30:3655-3662

Traherne JA, Horton R, Roberts AN et al (2006) Genetic analysis of completely sequenced disease-associated MHC haplotypes identifies shuffling of segments in recent human history. PLoS Genet 2(1):e9

Traherne JA, Martin M, Ward R et al (2010) Mechanisms of copy number variation and hybrid gene formation in the KIR immune gene complex. Hum Mol Genet 19(5):737-751

Willcox BE, Thomas LM, Bjorkman PJ (2003) Crystal structure of HLAA2 bound to LIR-1, a host and viral major histocompatibility complex receptor. Nat Immunol 4(9):913-919

Wiśniewski A, Wagner M, Nowak I et al (2013) 6.7-kbp deletion in LILRA3 (ILT6) gene is associated with later onset of the multiple sclerosis in a Polish population. Hum Immunol 74(3):353-357

Yang Z, Bjorkman PJ (2008) Structure of UL18, a peptide-binding viral MHC mimic, bound to a host inhibitory receptor. Proc Natl Acad Sci U S A 105(29):10095-10100

Yang ZH, Nielsen R, Goldman N, Pedersen AMK (2000) Codonsubstitution models for heterogeneous selection pressure at amino acid sites. Genetics 155:431-449

Zhang J, Kumar S, Nei M (1997) Small-sample tests of episodic adaptive evolution: a case study of primate lysozymes. Mol Biol Evol 14(12): $1335-1338$

Zhang Z, Li J, Zhao XQ, Wang J, Wong GK, Yu J (2006) KaKs_Calculator: calculating $\mathrm{Ka}$ and $\mathrm{Ks}$ through model selection and model averaging. Genomics ProteomE Bioinforma 4(4):259-263

Zuker M (2003) Mfold web server for nucleic acid folding and hybridization prediction. Nucleic Acids Res 31(13):3406-3415 